\title{
Conversion and possibility in syllogistic reasoning
}

\author{
LOUIS S. DICKSTEIN \\ Wellesley College, Wellesley, Massachusetts 02181
}

\begin{abstract}
Previous research on syllogistic reasoning has identified two bases of error on this task. One basis is the illicit conversion of universal affirmative and particular negative propositions. The second basis is the selection of propositional conclusions on indeterminate nonconversion syllogisms. The present study explores possible explanations for these phenomena. Data are presented that cast doubt on the explanation of conversion based upon the similarity between syllogistic premises and definitional statements. Even when the premises are modified so that they no longer resemble definitional statements, conversion still occurs. Bias toward symmetrical relations is discussed as an alternative explanation. Data are also presented to show that poor performance on indeterminate nonconversion syllogisms in large part reflects the failure of subjects to differentiate between possible and necessary conclusions. When this issue is clarified, performance significantly improves.
\end{abstract}

Recent research on syllogistic reasoning has produced a consensus that the illicit conversion of universal affirmative and particular negative propositions is an important contributor to errors on this task. Illicit conversion was first noted by Wilkins (1928) but was neglected for many years, as the atmosphere explanation (Woodworth \& Sells, 1935) dominated the literature. In 1959, Chapman and Chapman proposed illicit conversion together with probabilistic inference as an alternative to the atmosphere explanation. Chapman and Chapman (1959) identified 13 invalid syllogisms for which the dominant error followed logically from erroneously converted premises. Since then, a number of research findings have accumulated that support the construct of conversion (e.g., Ceraso \& Provitera, 1971; Dickstein, 1975; Erickson, 1974, 1978; Griggs, 1978; Revlin, Ammerman, Petersen, \& Leirer, 1978; Revlin \& Leirer, 1978).

As a result of these findings, conversion has been incorporated into most contemporary models of syllogistic reasoning (e.g., Dickstein, 1978; Erickson, 1978; Revlin \& Leirer, 1978). Unfortunately, while the principle of conversion has been widely accepted as a description of subject performance, there is less certainty about the explanation for conversion. One explanation, suggested by Chapman and Chapman (1959), is that the subject is misled into conversion of universal affirmative propositions by the similarity of such propositions to definitional statements. Thus, Chapman and Chapman (1959) note that a proposition such as "All A are B" resembles a definitional statement such as "All right angles are 90-deg angles." Because of this similarity, it is argued that the subject treats the syllogistic proposition as if it were a definitional statement and this leads to an identity interpretation.

One way of testing the validity of this explanation of conversion errors is to contrast the traditional format of syllogistic propositions with an alternative format containing modified propositions that still allow identity interpretations but are dissimilar to definitional statements. Such an alternative format might be constructed by modifying the syllogistic premises to emphasize the meaning of these premises as statements about class membership. Thus, the premise "All A are B" might be rewritten as "All members of the class $A$ are members of the class B." Such a formulation makes clear that there are two distinct classes here and that the issue concerns the relationship between membership in one class and membership in the other class. Such a statement bears little resemblance to a definitional statement. At the same time, it allows both identity and subset interpretations. If the similarity to definitional statements is responsible for illicit conversion of universal affirmative propositions, then there should be less conversion with the modified premises that no longer resemble definitional statements.

While conversion is an important factor in syllogistic reasoning, it is not the only factor responsible for errors. Indeed, Chapman and Chapman (1959) noted that conversion could account for errors on only 13 of the 45 invalid syllogisms included in the traditional syllogistic task. On the remaining 32 invalid syllogisms, subjects often draw propositional conclusions even though such conclusions are unjustified. This tendency cannot be explained by conversion because on these syllogisms, even after the premises are converted, propositional conclusions remain unjustified. The syllogisms are still indeterminate. While most research demonstrating this phenomenon has been conducted with a selection paradigm in which subjects are presented the major and minor premises and then select a conclusion from five response alternatives, Erickson (1978) has reported 
that it also holds for an evaluation paradigm in which the premises and the conclusion are presented and the subject judges the correctness of the conclusion.

The distinction proposed by Chapman and Chapman (1959) between invalid conversion and nonconversion syllogisms has been supported by research. Thus, Dickstein $(1975,1976)$ has shown that invalid conversion syllogisms are consistently more difficult than invalid nonconversion syllogisms. It may also be noted that errors on these syllogisms are not random, and a number of mechanisms have been proposed to account for the specific error choices (Chapman \& Chapman, 1959; Dickstein, 1978; Erickson, 1974; Revlis, 1975).

Revlin (Revlin \& Leirer, 1978; Revlis, 1975) has suggested that the primary source of error on invalid nonconversion syllogisms is a bias against nonpropositional conclusions. However, the reason for such a bias remains unclear. One possibility proposed by Revlis (1975) seems incorrect. Revlis (1975) suggested that the bias results from the imbalance between the 19 valid (determinate) and 45 invalid (indeterminate) syllogisms that constitute the traditional syllogistic task. According to this explanation, subjects do not expect so many invalid syllogisms, and, therefore, they avoid nonpropositional conclusions. Thus, the bias is viewed as an artifact of the task environment. However, this is an untenable explanation, as Dickstein (1976) has demonstrated that the avoidance of nonpropositional conclusions is unaffected by manipulation of the proportions of valid and invalid syllogisms. Performance on the invalid nonconversion syllogisms remains unchanged even when valid syllogisms constitute the majority of the syllogism set.

This paper proposes an alternative explanation for errors on invalid nonconversion syllogisms that is based upon the distinction between necessary and possible conclusions. A distinguishing feature of deductive reasoning is that a justifiable conclusion is one that is compelled by the premises. Indeed, strictly speaking, a deductive conclusion does not provide any new information that is not already contained in the premises. A conclusion that is merely possible but not necessitated by the premises is not acceptable under the rules of deductive reasoning. It is proposed that this distinguishing feature of deductive reasoning is not fully appreciated by subjects and that this may be responsible for the erroneous acceptance of propositional conclusions. ${ }^{1}$ In everyday thinking, it is unlikely that subjects impose such a strict constraint upon inferences that may be drawn. Indeed, a distinguishing feature of human thought may be that it seeks to move beyond established information toward new implications and inferences (see Dickstein, 1980). Subjects may be unfamiliar with the novel constraint of deductive reasoning and may therefore employ a less rigorous criterion of evidence. If this is the case, then subjects who are carefully instructed in this feature of deductive reasoning should perform better on invalid nonconversion syllogisms than subjects who are not sensitized to this issue.
In summary, this study addresses two separate issues in syllogistic reasoning. The first issue is the illicit conversion of premises. The definitional statement explanation of conversion is tested by comparing two premise formats. The second issue is the avoidance of nonpropositional conclusions on invalid nonconversion syllogisms. An explanation based upon the distinction between possible and necessary conclusions is tested by manipulating the instructions provided to subjects.

\section{METHOD}

Subjects were 56 female undergraduates at Wellesley College who participated in the study to meet a course requirement. Subjects were run in groups, and there was a female experimenter for all sessions.

There were two between-subjects factors (premise format and instructions), with two levels of each, and one withinsubjects factor (syllogism type) with three levels. One level of premise format consisted of the traditional premises. In this condition, all premises and possible conclusions were written in the classical syllogistic form (e.g., "All A are B"). The other level consisted of modified premises in which the class membership meaning of the premises was made explicit. In this condition, all premises and possible conclusions were written in an extended form (e.g., "All members of Class A are members of Class B"). One level of instructions ${ }^{2}$ did not contain any reference to the principle of necessity in drawing a conclusion from the premises. The other level of instructions included an additional paragraph that clarified that a propositional conclusion could be drawn only if the conclusion was compelled by the information in the premises. The three levels of syllogism type were valid, invalid conversion, and invalid nonconversion. Subjects were randomly assigned to the four between-subjects conditions, with 14 subjects in each condition.

All subjects received instructions clarifying the nature of the syllogistic task. Thus, subjects were informed that the word "some" means "at least some" and not "only some." The instructions were attached to the test booklet and subjects could refer to them at any time. The test booklet consisted of 16 pages of syllogisms with four syllogisms on each page. All subjects did the same 64 abstract syllogisms representing all premise combinations for each of the four figures of the syllogism ("figure" refers to the order of the terms in the major and minor premises). Nineteen of the syllogisms are valid and allow propositional conclusions. The remaining 45 are invalid and consist of 13 conversion syllogisms (see Dickstein, 1981) and 32 nonconversion syllogisms. Each syllogism was followed by five response alternatives, and the subject was required to select one alternative. These were "All S are P," "Some S are $P$," "No S are P," "Some S are not P," and "No valid conclusion" for the traditional format and an extended form of these alternatives for the modified format. These alternatives were always presented in the same order to avoid confusing the subjects. Similarly, the letters "S," "M," and "P" were always used to represent the subject, middle term, and predicate to avoid confusing the subjects and to facilitate reference to the instructions. Subjects completed all of the syllogisms at a single session, and no time limit was imposed.

\section{RESULTS}

The means and standard deviations for all groups for the percentage correct for valid, invalid conversion, and invalid nonconversion syllogisms are presented in Table 1 . The percentage correct data were analyzed with an analysis of variance for a three-factor mixed design 
Table 1

Means and Standard Deviations of Percentage Correct for All Conditions

\begin{tabular}{|c|c|c|c|c|c|c|}
\hline \multirow[b]{2}{*}{ Group } & \multicolumn{2}{|c|}{ Valid } & \multicolumn{2}{|c|}{$\begin{array}{c}\text { Invalid } \\
\text { Conversion }\end{array}$} & \multicolumn{2}{|c|}{$\begin{array}{c}\text { Invalid } \\
\text { Nonconversion }\end{array}$} \\
\hline & Mean & SD & Mean & SD & Mean & SD \\
\hline 1 & 60.5 & 18.2 & 37.3 & 27.7 & 71.9 & 22.6 \\
\hline 2 & 61.6 & 12.2 & 36.9 & 28.5 & 67.1 & 28.3 \\
\hline 3 & 63.1 & 19.4 & 35.2 & 29.7 & 57.1 & 29.6 \\
\hline 4 & 70.4 & 8.9 & 19.3 & 24.3 & 48.1 & 28.3 \\
\hline
\end{tabular}

Note-Group 1 received additional instructions and modified premises. Group 2 received additional instructions and traditional premises. Group 3 received control instructions and modified premises. Group 4 received control instructions and traditional premises.

with repeated measures on one factor. There were no significant main effects of premise format or instructions, but there was a main effect of syllogism type $[F(2,104)=53.3, p<.001]$. Performance was best on valid syllogisms (63.9\% correct), followed by invalid nonconversion syllogsims ( $61.0 \%$ correct), followed by invalid conversion syllogisms (32.2\% correct). There was also a significant interaction between instructions and syllogism type $[F(2,104)=5.76, p<.005]$. On valid syllogisms, performance was better without the additional instructions $(66.8 \%$ correct) than with the additional instructions $(61.1 \%$ correct), whereas for invalid conversion and invalid nonconversion syllogisms, performance was better with the additional instructions than without $(37.1 \%$ correct vs. $27.2 \%$ correct for invalid conversion syllogisms and $69.5 \%$ correct vs. $52.6 \%$ correct for invalid nonconversion syllogisms). There were no other significant interactions.

In order to further clarify the nature of the two significant findings, three additional three-factor mixeddesign analyses of variance were conducted using only two of the three syllogism types at a time. With regard to the main effect of syllogism type, these analyses showed that valid syllogisms and invalid nonconversion syllogisms were both significantly different from invalid conversion syllogisms, but not different from each other. With regard to the interaction between syllogism type and instructions, these analyses showed that the significant interaction was due to the difference between valid syllogisms and invalid nonconversion syllogisms. The interactions between syllogism type and instructions were not significant for the other two comparisons.

The improvement on invalid nonconversion syllogisms due to additional instructions was examined further by looking at the individual premise combinations that constitute this set of syllogisms. The 32 invalid nonconversion syllogisms consist of eight different premise combinations, each of which appears in all four figures (see Dickstein, 1978). Examination of the eight premise combinations revealed that the effect of instructions was consistent throughout the set. In all eight instances, mean performance of the groups that received additional instructions was better than that of the groups that did not receive these instructions. The two instruction conditions were also compared with regard to the pattern of errors on invalid nonconversion syllogisms. Across the 32 syllogisms, the groups that did not receive the additional instructions drew a total of 425 erroneous propositional conclusions. These included $6(1.4 \%)$ universal affirmative conclusions, 120 (28.2\%) particular affirmative conclusions, 104 (24.5\%) universal negative conclusions, and 195 (45.9\%) particular negative conclusions. The groups that received additional instructions drew a total of 274 erroneous propositional conclusions. These included $4(1.5 \%)$ universal affirmative conclusions, $75(27.4 \%)$ particular affirmative conclusions, $79(28.8 \%)$ universal negative conclusions, and 116 (42.3\%) particular negative conclusions. It is apparent that the distribution of errors was highly similar in the two conditions.

\section{DISCUSSION}

The main finding of this study is that clarification of the role of necessity in deductive inference significantly improves performance. As predicted, this improvement occurs primarily among the invalid nonconversion syllogisms. The degree of improvement is substantial, amounting to a mean difference of 5.4 correct responses out of a total of 32 .

These results support the argument that poor performance on indeterminate syllogisms reflects the failure of subjects to confine inferences to propositions that are compelled by the premises. Without clarification of this issue, subjects are ready to endorse conclusions that are merely possible, just as they do on reasoning problems with real-life content (see Dickstein, 1980). It is interesting to note that while there is a marked reduction in errors when this issue is clarified, the distribution of errors remains the same. This is not surprising, since the additional instructions do not address the issue of selection of particular erroneous propositional conclusions.

It is important to note that while clarification of the necessity issue improves performance, there remain a number of errors. One explanation for these continuing errors is that subjects may fail to recognize that the inference they wish to draw is only a possibility and that other relationships between the subject and the predicate are also consistent with the information in the premises. Erickson $(1974,1978)$ has emphasized the incomplete analysis of possibilities as a deficiency of subjects on this task. Alternatively, Dickstein (1978) has suggested several specific reasoning processes that might account for these errors. There is a need for more experimental research to test these formulations.

The second major finding of this study is that alteration of the form of the premises does not improve performance. More specifically, the modification of the traditional format of the premises into a format emphasizing class membership does not reduce the frequency of conversion errors. The 13 invalid conversion syllogisms remain just as difficult, with correct responses occurring less than $40 \%$ of the time.

These findings cast doubt on the suggestion of Chapman and Chapman (1959) that it is the similarity of the premises in the traditional syllogism to definitional statements that encourages subjects to encode universal affirmative propositions as identity relations. The modified class membership premises do not look at all like definitional statements. Nonetheless, subjects continue to respond in a manner reflecting illicit conversion. ${ }^{3}$

There is an alternative explanation for conversion that has also been discussed in the literature and does have some research support. This explanation focuses on the symmetry of the 
relationship that results after illicit conversion. An identity interpretation of universal affirmative propositions yields a symmetrical relation between the two terms, whereas a subset interpretation does not. Similarly, an overlap interpretation of particular negative propositions yields a symmetrical relation between the two terms, whereas a superset interpretation does not. This explanation has been discussed by Dickstein (1978), Henle (1978), and Revlin and Leirer (1978) among others. Recent evidence of a general tendency on the part of subjects to assume symmetrical relations between classes has been provided by Tsal (1977). Further research should be directed toward a clearer understanding of the origins of this cognitive propensity.

Finally, the present study provides further support for the differentiation of two subsets of invalid syllogisms: conversion syllogisms and nonconversion syllogisms (Chapman \& Chapman, 1959; Dickstein, 1975, 1976). Invalid conversion syllogisms are significantly more difficult than invalid nonconversion syllogisms. Furthermore, the significant difference in difficulty between valid and invalid syllogisms that has been reported previously (Dickstein, 1975, 1976) is primarily due to the extreme difficulty of invalid conversion syllogisms.

\section{REFERENCES}

Ceraso, J., \& Provitera, A. Sources of error in syllogistic reasoning. Cognitive Psychology, 1971, 2, 400-410.

Chapman, L., \& Chapman, J. Atmosphere effect reexamined. Journal of Experimental Psychology, 1959, 58, 220-226.

Dickstein, L. Effects of instructions and premise order on errors in syllogistic reasoning. Journal of Experimental Psychology: Human Learning and Memory, 1975, 1, 376-384.

Dickstern, L. Differential difficulty of categorical syllogisms. Bulletin of the Psychonomic Society, 1976, 8, 330-332.

Dickstein, L. Error processes in syllogistic reasoning. Memory \& Cognition, 1978, 6, 537-543.

Dickste in, L. Inference errors in deductive reasoning. Bulletin of the Psychonomic Society, 1980, 16, 414-416.

Dickstern, L. The meaning of conversion in syllogistic reasoning. Bulletin of the Psychonomic Society, 1981, 18, 135-138.

Erickson, J. A set analysis theory of behavior in formal syllogistic reasoning tasks. In $\mathbf{R}$. Solso (Ed.), Theories of cognitive psychology: The Loyola symposium. Hillsdale, N.J: Erlbaum, 1974.

ERICKson, J. Research on syllogistic reasoning. In R. Revlin \& R. Mayer (Eds.), Human reasoning. Washington, D.C: Winston-Wiley, 1978.

GRIGGS, R. Drawing inferences from set inclusion information given in text. In R. Revlin \& R. Mayer (Eds.), Human reasoning. Washington, D.C: Winston-Wiley, 1978.

Henle, M. Foreward. In R. Revlin \& R. Mayer (Eds.), Human reasoning. Washington, D.C: Winston-Wiley, 1978.

Revlin, R., Ammerman, K., Petersen, K., \& Leirer, V. Category relations and syllogistic reasoning. Journal of Educational Psychology, 1978, 70, 613-625.

REVLin, R., \& Leiren, V. The effects of personal biases on syllogistic reasoning: Rational decisions from personalized representations. In R. Revlin \& R. Mayer (Eds.), Human reasoning. Washington, D.C: Winston-Wiley, 1978.

REvLIs, R. Syllogistic reasoning: Logical decisions from a complex data base. In R. Falmagne (Ed.), Reasoning: Representation and process. Hillsdale, N.J: Erlbaum, 1975.

Tsal, Y. Symmetry and transitivity assumptions about a nonspecified logical relation. Quarterly Journal of Experimental Psychology, 1977, 29, 677-684.

Wilkins, M. The effect of changed material on ability to do formal syllogistic reasoning. Archives of Psychology, 1928, 16, $1-83$.

Woodworth, R., \& Sells, S. An atmosphere effect in formal syllogistic reasoning. Journal of Experimental Psychology, $1935,18,451-460$.

\section{NOTES}

1. This argument is different from the construct of probabilistic inference proposed by Chapman and Chapman (1959). Probabilistic inference is a much more specific mechanism that generates predictions about the particular erroneous nonpropositional conclusions selected by subjects. See Dickstein (1978) for a critique of probabilistic inference.

2. Both sets of instructions are available upon request from the author.

3. Invalid conversion syllogisms actually involve two different kinds of illicit conversion. In most instances, the illicit conversion involves a universal affirmative proposition. In some instances, it involves a particular negative proposition. Since the similarity to definitional statements holds only for universal affirmative propositions, the data were reanalyzed using only those invalid conversion syllogisms that involved universal affirmative propositions. There were no differences in the findings.

(Received for publication September 2, 1981.) 